\title{
STOCHASTIC CONTROLLABILITY OF SYSTEMS WITH MULTIPLE DELAYS IN CONTROL
}

\author{
JERZY KLAMKA \\ Institute of Control Engineering \\ Silesian University of Technology, Akademicka 16, 44-100 Gliwice, Poland \\ e-mail: jerzy.klamka@polsl.pl
}

\begin{abstract}
Finite-dimensional stationary dynamic control systems described by linear stochastic ordinary differential state equations with multiple point delays in control are considered. Using the notation, theorems and methods used for deterministic controllability problems for linear dynamic systems with delays in control as well as necessary and sufficient conditions for various kinds of stochastic relative controllability in a given time interval are formulated and proved. It will be proved that, under suitable assumptions, relative controllability of an associated deterministic linear dynamic system is equivalent to stochastic relative exact controllability and stochastic relative approximate controllability of the original linear stochastic dynamic system. As a special case, relative stochastic controllability of dynamic systems with a single point delay is also considered. Some remarks and comments on the existing results for stochastic controllability of linear dynamic systems are also presented.
\end{abstract}

Keywords: controllability, linear control systems, stochastic control systems, delayed controls, multiple delays.

\section{Introduction}

Controllability is one of the fundamental concepts in mathematical control theory and plays an important role in both deterministic and stochastic control theories (Klamka, 1991; 1993; Mahmudov, 2003a; Mahmudov and Denker, 2000). Controllability is a qualitative property of dynamic control systems and is of particular importance in control theory. A systematic study of controllability started at the beginning of the 1960s, when the theory of controllability based on the description in the form of a state space for both time-invariant and time-varying linear control systems was worked out. Roughly speaking, controllability generally means that it is possible to steer a dynamic control system from an arbitrary initial state to an arbitrary final state using a set of admissible controls. In the literature there are many different definitions of controllability, for both linear (Klamka, 1991; 1993; Mahmudov, 2001a; Mahmudov and Denker, 2000) and nonlinear dynamic systems (Klamka, 2000; Mahmudov, 2002; 2003b; Mahmudov and Zorlu, 2003), which strongly depend on the class of dynamic control systems and the set of admissible controls (Klamka, 1991; 1996). Therefore, for deterministic linear and nonlinear dynamic systems there exist many different necessary and sufficient conditions for global and local controllability (Klamka, 1991; 1993; 1996; 2000).

In recent years various controllability problems for different types of linear dynamical systems have been considered in many publications. Extensive lists of these can be found, e.g., in the monograph (Klamka, 1991) or in the survey papers (Klamka, 1993; 1996; 2000). However, it should be stressed that most literature in this field has been mainly concerned with deterministic controllability problems for finite-dimensional linear dynamic systems with unconstrained controls and without delays.

For stochastic linear and nonlinear control systems the situation is less satisfactory. In recent years extensions of deterministic controllability concepts to stochastic control systems have been discussed only in rather few publications. In the papers (Bashirov and Kerimov, 1997; Bashirov and Mahmudov, 1991; Erhard and Kliemenn, 1982; Mahmudov, 2001a; Mahmudov and Denker, 2000; Zabczyk, 1991), different kinds of stochastic controllability were discussed for linear finite dimensional stationary and nonstationary control systems. The papers (Fernandez-Cora et al., 1999; Kim, 2004; Mahmudov, $2001 b$; 2003a) are devoted to a systematic study of approximate and exact stochastic controllability for linear infinite dimensional control systems defined in Hilbert 
spaces. Stochastic controllability for finite dimensional nonlinear stochastic systems was discussed in (Arapostathis et al., 2001; Mohamed and Zorlu, 2003; Sunahara et al., 1974; 1975). Using the theory of bounded nonlinear operators and linear semigroups, different types of stochastic controllability for nonlinear infinite dimensional control systems defined in Hilbert spaces were considered in (Mahmudov, 2002; 2003b). In the papers (Klamka and Socha, 1974; 1980), Lyapunov techniques were used to formulate and prove sufficient conditions for stochastic controllability of nonlinear finite dimensional stochastic systems with point delays in the state variable. Moreover, it should be pointed out that the functional analysis approach to stochastic controllability problems was also extensively discussed for both linear and nonlinear stochastic control systems in (Fernandez-Cora et al., 1999; Kim, 2004; Mahmudov, 2001b; 2002; 2003a; Subramaniam and Balachandran, 2002).

In the present paper we shall study stochastic controllability problems for linear dynamic systems, which are natural generalizations of controllability concepts well known in infinite dimensional control systems theory (Klamka, 1991; 1993). More precisely, we shall consider stochastic relative exact and approximate controllability problems for finite-dimensional linear stationary dynamical systems with multiple constant point delays in control described by stochastic ordinary differential state equations. More precisely, using techniques similar to those presented in the papers (Mahmudov, 2001a; 2001b; Mahmudov and Denker, 2000), we shall formulate and prove necessary and sufficient conditions for stochastic relative exact controllability in a prescribed time interval for linear stationary stochastic dynamic systems with multiple constant point delays in control.

Roughly speaking, it will be proved that, under suitable assumptions, relative controllability of an associated deterministic linear dynamic system is equivalent to stochastic relative exact controllability and stochastic relative approximate controllability of the original linear stochastic dynamic system. This is a generalization to the control delayed case of some previous results concerning stochastic controllability of linear dynamic systems without delays in control (Mahmudov, 2001a; 2001b; Mahmudov and Denker, 2000).

The paper is organized as follows: Section 2 contains a mathematical model of a linear, stationary stochastic dynamical system with multiple constant point delays in the control. Moreover, in this section some basic notation and definitions of stochastic relative exact and stochastic approximate controllability are presented. Also, some preliminary results are included. In Section 3, using results and methods taken directly from deterministic controllability problems, necessary and sufficient conditions for exact and approximate stochastic relative controllability are formulated and proved. In Section 4, as a special case, relative stochastic controllability in a given time interval of dynamic systems with single point delays is also considered. Finally, Section 5 contains concluding remarks and states some open controllability problems for more general stochastic dynamic systems.

\section{System description}

Throughout this paper, unless otherwise specified, we use the following standard notation: Let $(\Omega, F, P)$ be a complete probability space with probability measure $P$ on $\Omega$ and a filtration $\left\{F_{t} \mid t \in[0, T]\right\}$ generated by an $n$ dimensional Wiener process $\{w(s): 0 \leq s \leq t\}$ defined on the probability space $(\Omega, F, P)$, (Zabczyk, 1991).

Let $L_{2}\left(\Omega, F_{T}, \mathbb{R}^{n}\right)$ denote the Hilbert space of all $F_{T}$-measurable square integrable random variables with values in $\mathbb{R}^{n}$. Moreover, let $L_{2}^{F}\left([0, T], \mathbb{R}^{n}\right)$ denote the Hilbert space of all square integrable and $F_{t}$-measurable processes with values in $\mathbb{R}^{n}$

In the theory of linear, finite-dimensional, timeinvariant stochastic dynamical control systems we use the mathematical model given by the following stochastic ordinary differential state equation with multiple point delays in control:

$$
\mathrm{d} x(t)=\left(A x(t)+\sum_{i=0}^{i=M} B_{i} u\left(t-h_{i}\right)\right) \mathrm{d} t+\sigma \mathrm{d} w(t)
$$

for $t \in[0, T]$, with the initial conditions

$$
x(0)=x_{0} \in L_{2}\left(\Omega, F_{T}, \mathbb{R}^{n}\right) \text { and } u(t)=0
$$

for $t \in\left[-h_{M}, 0\right)$, where the state $x(t) \in \mathbb{R}^{n}=X$ and the control $u(t) \in \mathbb{R}^{m}=U, A$ is an $n \times n$ dimensional constant matrix, $B_{i}, i=0,1,2, \ldots, M$, are $n \times m$ dimensional constant matrices, $\sigma$ is an $n \times n$ dimensional constant matrix, and $0=h_{0}<h_{1}<\cdots<h_{i}<\cdots<$ $h_{M-1}<h_{M}$ are constant delays.

In the sequel, for simplicity of discussion, we generally assume that the set of admissible controls has the form $U_{a d}=L_{2}^{F}\left([0, T], \mathbb{R}^{m}\right)$. Moreover, it is well known (Mahmudov, 2001a; 2001b; Mahmudov and Denker, 2000; Mahmudov and Zorlu, 2003), that for the given initial conditions (2) and any admissible control $u \in U_{a d}$, for $t \in[0, T]$ there exists a unique solution $x\left(t ; x_{0}, u\right) \in$ $L_{2}\left(\Omega, F_{t}, \mathbb{R}^{n}\right)$ of the linear stochastic differential state equation (1), which can be represented in the following integral form:

$$
\begin{aligned}
x\left(t ; x_{0}, u\right)= & \exp (A t) x_{0} \\
& +\int_{0}^{t} \exp (A(t-s))\left(\sum_{i=0}^{i=M} B_{i} u\left(s-h_{i}\right)\right) \mathrm{d} s \\
& +\int_{0}^{t} \exp (A(t-s)) \sigma \mathrm{d} w(s) .
\end{aligned}
$$


Thus, without loss of generality, taking into account the zero initial control for $t \in\left[-h_{M}, 0\right]$ and changing the order of integration, the solution $x\left(t ; x_{0}, u\right)$ for $h_{k}<t \leq$ $h_{k+1}, k=0,1,2, \ldots, M-1, t \in[0, h]$ has the following form, which is more convenient for further deliberations (Klamka, 1991):

$$
\begin{aligned}
x\left(t ; x_{0}, u\right)= & \exp (A t) x_{0} \\
& +\sum_{i=0}^{i=k} \int_{0}^{t-h_{i}} \exp \left(A\left(t-s-h_{i}\right)\right) B_{i} u(s) \mathrm{d} s \\
& +\int_{0}^{t} \exp (A(t-s)) \sigma \mathrm{d} w(s) .
\end{aligned}
$$

Moreover, for $t>h_{M}$ we have

$$
\begin{aligned}
x\left(t ; x_{0}, u\right)= & \exp (A t) x_{0} \\
& +\sum_{i=0}^{i=M} \int_{0}^{t-h_{i}} \exp \left(A\left(t-s-h_{i}\right)\right) B_{i} u(s) \mathrm{d} s \\
& +\int_{0}^{t} \exp (A(t-s)) \sigma \mathrm{d} w(s)
\end{aligned}
$$

or, equivalently, for $h_{k}<t<h_{k+1}$ and for $k=$ $0,1, \ldots, M-1$,

$$
\begin{aligned}
& x\left(t ; x_{0}, u\right) \\
& =\exp (A t) x_{0} \\
& +\sum_{i=0}^{i=k-1} \int_{t-h_{i+1}}^{t-h_{i}}\left(\sum_{j=0}^{j=i} \exp \left(A\left(t-s-h_{j}\right)\right) B_{j}\right) \\
& \quad \cdot u(s) \mathrm{d} s \\
& +\int_{0}^{t-h_{k}}\left(\sum_{j=0}^{j=k} \exp \left(A\left(t-s-h_{j}\right)\right) B_{j}\right) u(s) \mathrm{d} s \\
& +\int_{0}^{t} \exp (A(t-s)) \sigma \mathrm{d} w(s) .
\end{aligned}
$$

Similarly, for $t>h_{M}$,

$$
\begin{aligned}
& x\left(t ; x_{0}, u\right) \\
& =\exp (A t) x_{0} \\
& +\sum_{i=0}^{i=k-1} \int_{t-h_{i+1}}^{t-h_{i}}\left(\sum_{j=0}^{j=i} \exp \left(A\left(t-s-h_{j}\right)\right) B_{j}\right) \\
& \quad \cdot u(s) \mathrm{d} s \\
& +\int_{0}^{t-h_{M}}\left(\sum_{j=0}^{j=M} \exp \left(A\left(t-s-h_{j}\right)\right) B_{j}\right) u(s) \mathrm{d} s \\
& +\int_{0}^{t} \exp (A(t-s)) \sigma \mathrm{d} w(s) .
\end{aligned}
$$

Now, for a given final time $T$, using the form of the integral solution $x\left(t ; x_{0}, u\right)$, let us introduce operators and sets which will be used in next parts of the paper (Klamka, 1991).

First of all, for $h_{k}<T<h_{k+1}$ and for $k=$ $0,1, \ldots, M-1$, we define the following linear and bounded control operator $L_{T} \in L_{2}^{F}\left([0, T], \mathbb{R}^{m}\right) \rightarrow$ $L_{2}\left(\Omega, F_{T}, \mathbb{R}^{n}\right)$ :

$$
\begin{aligned}
& L_{T} u \\
& =\sum_{i=0}^{i=k-1} \int_{T-h_{i+1}}^{T-h_{i}}\left(\sum_{j=0}^{j=i} \exp \left(A\left(T-s-h_{j}\right)\right) B_{j}\right) \\
& \quad \cdot u(s) \mathrm{d} s \\
& \left.\quad+\int_{0}^{T-h_{k}}\left(\sum_{j=0}^{j=k} \exp A\left(T-s-h_{j}\right)\right) B_{j}\right) u(s) \mathrm{d} s .
\end{aligned}
$$

Moreover, for $T>h_{M}$ we have

$$
\begin{aligned}
& L_{T} u \\
& =\sum_{i=0}^{i=M-1} \int_{T-h_{i+1}}^{T-h_{i}}\left(\sum_{j=0}^{j=i} \exp \left(A\left(T-s-h_{j}\right)\right) B_{j}\right) \\
& \quad \cdot u(s) \mathrm{d} s \\
& \quad+\int_{0}^{T-h_{M}}\left(\sum_{j=0}^{j=M} \exp \left(A\left(T-s-h_{j}\right)\right) B_{j}\right) u(s) \mathrm{d} s
\end{aligned}
$$

and its adjoint linear and bounded operator is $L_{T}^{*} \in$ $L_{2}\left(\Omega, F_{T}, \mathbb{R}^{n}\right) \rightarrow L_{2}^{F}\left([0, T], \mathbb{R}^{m}\right)$,

$$
L_{T}^{*} z=\left\{\begin{array}{r}
B_{0}^{*} \exp \left(A^{*}(T-t)\right) E\left\{z \mid F_{t}\right\} \\
\text { for } t \in\left[0, T-h_{M}\right], \\
\left(\sum_{j=1}^{j=i} B_{j}^{*} \exp \left(A^{*}\left(T-t-h_{j}\right)\right)\right) E\left\{z \mid F_{t}\right\} \\
\text { for } \quad t \in\left(T-h_{i+1}, T-h_{i}\right], \\
i=0,1, \ldots, M-1,
\end{array}\right.
$$

where the asterisk denotes the adjoint operator or the matrix transpose.

From the above notation it follows that the set of all states reachable from the initial state $x(0)=x_{0} \in$ $L_{2}\left(\Omega, F_{T}, \mathbb{R}^{n}\right)$ in time $T>0$ using admissible controls has the form

$$
\begin{aligned}
& R_{T}\left(U_{a d}\right) \\
& =\left\{x\left(T ; x_{0}, u\right) \in L_{2}\left(\Omega, F_{T}, \mathbb{R}^{n}\right): u \in U_{a d}\right\} \\
& =\exp (A t) x_{0}+\operatorname{Im} L_{T}+\int_{0}^{T} \exp (A(T-s)) \sigma \mathrm{d} w(s) .
\end{aligned}
$$

Moreover, we introduce the concept of the linear controllability operator (Klamka, 1991; 1993; Mahmudov, 2001a; Mahmudov and Denker, 2000) $C_{T} \in$ 
$L\left(L_{2}\left(\Omega, F_{T}, \mathbb{R}^{n}\right), L_{2}\left(\Omega, F_{T}, \mathbb{R}^{n}\right)\right)$, which is strongly associated with the control operator $L_{T}$ and is defined by the following equality:

$$
\begin{aligned}
C_{T}= & L_{T} L_{T}^{*} \\
= & \sum_{i=0}^{i=k-1} \int_{T-h_{i+1}}^{T-h_{i}}\left(\sum_{j=0}^{j=i} \exp \left(A\left(T-t-h_{j}\right)\right) B_{j}\right) \\
& \left(\sum_{j=0}^{j=i} B_{j}^{*} \exp \left(A^{*}\left(T-t-h_{j}\right)\right)\right) E\left\{\cdot \mid F_{t}\right\} \mathrm{d} t \\
+ & \int_{0}^{T-h_{k}}\left(\sum_{j=0}^{j=k} \exp \left(A\left(T-t-h_{j}\right)\right) B_{j}\right) \\
& \left(\sum_{j=0}^{j=k} B_{j}^{*} \exp \left(A^{*}\left(T-t-h_{j}\right)\right)\right) E\left\{\cdot \mid F_{t}\right\} \mathrm{d} t
\end{aligned}
$$

for $h_{i+1}<T<h_{i}, i=0,1, \ldots, M-1$, and for $T>$ $h_{M}$ by

$$
\begin{aligned}
C_{T}= & L_{T} L_{T}^{*} \\
= & \sum_{i=0}^{i=M-1} \int_{T-h_{i+1}}^{T-h_{i}}\left(\sum_{j=0}^{j=i} \exp \left(A\left(T-t-h_{j}\right)\right) B_{j}\right) \\
& \left(\sum_{j=0}^{j=i} B_{j}^{*} \exp \left(A^{*}\left(T-t-h_{j}\right)\right)\right) E\left\{\cdot \mid F_{t}\right\} \mathrm{d} t \\
+ & \int_{0}^{T-h_{M}}\left(\sum_{j=0}^{j=M} \exp \left(A\left(T-t-h_{j}\right)\right) B_{j}\right) \\
& \left(\sum_{j=0}^{j=M} B_{j}^{*} \exp \left(A^{*}\left(T-t-h_{j}\right)\right)\right) E\left\{\cdot \mid F_{t}\right\} \mathrm{d} t .
\end{aligned}
$$

Finally, let us recall the $n \times n$-dimensional deterministic controllability matrix (Klamka, 1991) for $h_{i+1}<$ $T<h_{i}, i=0,1, \ldots, M-1$,

$$
\begin{aligned}
G_{T}= & L_{T} L_{T}^{*} \\
= & \sum_{i=0}^{i=k-1} \int_{T-h_{i+1}}^{T-h_{i}}\left(\sum_{j=0}^{j=i} \exp \left(A\left(T-t-h_{j}\right)\right) B_{j}\right) \\
& \left(\sum_{j=0}^{j=i} B_{j}^{*} \exp \left(A^{*}\left(T-t-h_{j}\right)\right)\right) \mathrm{d} t \\
& +\int_{0}^{T-h_{k}}\left(\sum_{j=0}^{j=k} \exp \left(A\left(T-t-h_{j}\right)\right) B_{j}\right) \\
& \left(\sum_{j=0}^{j=k} B_{j}^{*} \exp \left(A^{*}\left(T-t-h_{j}\right)\right)\right) \mathrm{d} t
\end{aligned}
$$

and for $T>h_{M}$

$$
\begin{aligned}
G_{T}= & L_{T} L_{T}^{*} \\
= & \sum_{i=0}^{i=M-1} \int_{T-h_{i+1}}^{T-h_{i}}\left(\sum_{j=0}^{j=i} \exp \left(A\left(T-t-h_{j}\right)\right) B_{j}\right) \\
& \left(\sum_{j=0}^{j=i} B_{j}^{*} \exp \left(A^{*}\left(T-t-h_{j}\right)\right)\right) \mathrm{d} t \\
+ & \int_{0}^{T-h_{M}}\left(\sum_{j=0}^{j=M} \exp \left(A\left(T-t-h_{j}\right)\right) B_{j}\right) \\
& \left(\sum_{j=0}^{j=M} B_{j}^{*} \exp \left(A^{*}\left(T-t-h_{j}\right)\right)\right) \mathrm{d} t .
\end{aligned}
$$

It is well known that in the theory of dynamic systems with delays in control or in state variables, it is necessary to distinguish between two fundamental concepts of controllability, namely, relative controllability and absolute controllability (Klamka, 1991; 1993; 2000). In this paper we shall concentrate on the weaker concept of relative controllability on a given time interval $[0, T]$. On the other hand, since for the stochastic dynamic system (1) the state space $L_{2}\left(\Omega, F_{t}, \mathbb{R}^{n}\right)$ is in fact an infinitedimensional space, we distinguish exact, or strong, controllability and approximate, or weak, controllability. Using the notation given above for the stochastic dynamic system (1), we define the following concepts of stochastic relative exact and stochastic relative approximate controllability (Klamka, 1991; Mahmudov, 2001a; Mahmudov and Denker, 2000).

Definition 1. The stochastic dynamical system (1) is said to be stochastically relatively exactly controllable on $[0, T]$ if $R_{T}\left(U_{a d}\right)=L_{2}\left(\Omega, F_{T}, \mathbb{R}^{n}\right)$.

Definition 2. The stochastic dynamical system (1) is said to be stochastically relatively approximately controllable on $[0, T]$ if $\overline{R_{T}\left(U_{a d}\right)}=L_{2}\left(\Omega, F_{T}, \mathbb{R}^{n}\right)$.

Remark 1. Since the state space $L_{2}\left(\Omega, F_{T}, \mathbb{R}^{n}\right)$ is infinite dimensional, it is necessary to distiguish between exact and approximate controllability. Moreover, from Definitions 1 and 2 it directly follows that stochastic relative exact controllability is generally a stronger concept than stochastic relative approximate controllability. However, there are many cases when these two concepts coincide.

Remark 2. Since the stochastic dynamical system (1) is linear and there are no constraints on control values, without loss of generality in the above two definitions it is enough to take the zero initial condition $x_{0}=0 \in$ $L_{2}\left(\Omega, F_{T}, \mathbb{R}^{n}\right)$. 
Remark 3. It should be pointed out that in the case of delayed controls the above controllability concepts essentially depend on the length of the time interval $[0, T]$.

Remark 4. Let us observe that for a final time $T \leq h_{1}$ the stochastic dynamical system (1) is in fact a system without delays.

Remark 5. Since the dynamical system (3) is stationary, the controllability matrix GT(s) has the same rank at least for all $s \in[0, T-h k]$, if $h_{k+1}<T<h_{k}$, $k=0,1, \ldots, M-1$, or for $s \in\left[0, T-h_{M}\right]$, if $T>h_{M}$, (Klamka, 1991).

Remark 6. From the form of the controllability operator $C_{T}$ it immediately follows that this operator is selfadjoint.

Remark 7. In this paper only the relative stochastic controllability of the dynamic system (1) is considered, and therefore the definitions for absolute approximate or exact stochastic controllability are omitted.

In the sequel we study the relationship between the controllability concepts for the stochastic dynamic system (1) and the controllability of the associated deterministic dynamic system with multiple delays in control of the form

$$
y^{\prime}(t)=A y(t)+\sum_{i=0}^{i=M} B_{i} v\left(t-h_{i}\right), \quad t \in[0, T],
$$

where the admissible controls $v \in L_{2}\left([0, T], \mathbb{R}^{m}\right)$.

For the deterministic system (3) let us denote by $R_{T}$ the set of all states reachable from the initial state $y(0)=$ 0 in time $T>0$ using admissible controls.

Definition 3. (Klamka, 1991) The deterministic dynamic system (3) is said to be relatively controllable on $[0, T]$ if $R_{T}=\mathbb{R}^{n}$.

Remark 8. Since the dynamic system (3) is finite dimensional, relative approximate controllability and relative exact controllability coincide, and we have only one definition of relative controllability.

Now, for completeness of discussion, let us recall a lemma concerning relative controllability of the deterministic system (3).

Lemma 1. (Klamka, 1991) The following conditions are equivalent:

(i) The deterministic system (3) is relatively controllable on $[0, T]$.

(ii) The controllability matrix $G_{T}$ is nonsingular. (iii) There holds

$$
\begin{aligned}
\operatorname{rank}[ & B_{0}, B_{1}, \ldots, B_{k}, \ldots, B_{M}, \\
& A B_{0}, A B_{1}, \ldots, A B_{k}, \ldots, A B_{M}, \ldots, \\
& A^{n-1} B_{0}, A^{n-1} B_{1}, \ldots, A^{n-1} B_{k}, \ldots, \\
& \left.A^{n-1} B_{M}\right]=n .
\end{aligned}
$$

Now, let us formulate an auxiliary lemma excerpted directly from the theory of stochastic processes which will be used in the sequel in the proofs of the main results.

Lemma 2. (Mahmudov, 2001a; Mahmudov and Denker, 2000; Mahmudov and Zorlu, 2003) For every $z \in L_{2}\left(\Omega, F_{T}, \mathbb{R}^{n}\right)$, there exists a process $q \in$ $L_{2}^{F}\left([0, T], \mathbb{R}^{n \times n}\right)$ such that

$$
C_{T} z=G_{T} E z+\int_{0}^{T} G_{T}(s) q(s) \mathrm{d} w(s) .
$$

Taking into account the above notation, definitions and lemmas, in the next section we shall formulate and prove conditions for stochastic relative exact and stochastic relative approximate controllability for the stochastic dynamic system (1).

\section{Stochastic relative controllability}

In this section, using the lemmas given in Section 2, we shall formulate and prove the main result of the paper, which says that stochastic relative exact and, in consequence, also approximate controllability of the stochastic system (1) are in fact equivalent to relative controllability of the associated linear deterministic system (3).

Theorem 1. The following conditions are equivalent:

(i) The deterministic system (3) is relatively controllable on $[0, T]$.

(ii) The stochastic system (1) is stochastically relatively exactly controllable on $[0, T]$.

(iii) The stochastic system (1) is stochastically relatively approximately controllable on $[0, T]$.

Proof. $\quad(i) \Rightarrow($ ii $)$ Let us assume that the deterministic system (3) is relatively controllable on $[0, T]$. Then, it is well known (Klamka, 1991; 1993; Klamka and Socha, 1977) that the symmetric relative deterministic controllability matrix $G_{T}(s)$ is invertible and strictly positive definite at least for all $s \in\left[0, T-h_{k}\right]$, if $h_{k}<T<h_{k+1}$, $k=0,1,2, \ldots, M-1$ or at least for all $s \in\left[0, T-h_{M}\right]$ if $T>h_{M}$, (Klamka, 1991). Hence, for some $\gamma>0$, we have

$$
\left\langle G_{T}(s) x, x\right\rangle \geq \gamma\|x\|^{2}
$$

for all $s \in\left[0, T-h_{M}\right]$ and for all $x \in \mathbb{R}_{n}$. In order to prove stochastic relative exact controllability on $[0, T]$ for 
the stochastic system (1), we use the relationship between the controllability operator $C_{T}$ and the controllability matrix $G_{T}$ given in Lemma 2, to express $E\left\langle C_{T} z, z\right\rangle$ in terms of $\left\langle G_{T} E z, E z\right\rangle$. First of all, we obtain

$$
\begin{aligned}
E\left\langle C_{T} z, z\right\rangle & E\left\langle G_{T} E z+\int_{0}^{T} G_{T}(s) q(s) \mathrm{d} w(s), E z\right. \\
& \left.+\int_{0}^{T} q(s) \mathrm{d} w(s)\right\rangle \\
= & \left\langle G_{T} E z, E z\right\rangle+E \int_{0}^{T}\left\langle G_{T}(s) q(s), q(s)\right\rangle \mathrm{d} s \\
\geq & \gamma\left(\|E z\|^{2}+E \int_{0}^{T}\|q(s)\|^{2} \mathrm{~d} s\right)=\gamma E\|z\|^{2} .
\end{aligned}
$$

Hence, in the operator sense, we have $C_{T} \geq \gamma I$, which means that the operator CT is strictly positive definite, and thus that the inverse linear operator $C_{T}^{-1}$ is bounded. Therefore, the stochastic relative exact controllability on $[0, T]$ of the stochastic dynamic system (1) directly follows from the results given in (Klamka, 1991).

(ii) $\Rightarrow($ iii $)$ This implication is obvious (Klamka, 1991; Mahmudov, 2001b; 2002; 2003a).

$($ iii $) \Rightarrow(i)$ Assume that the stochastic dynamic system (1) is stochastically relatively approximately controllable on $[0, T]$, and hence its linear self-adjoint controllability operator is positive definite, i.e., $C_{T}>0$ (Klamka, 1991). Then, using the resolvent operator $R\left(\lambda, C_{T}\right)$ and following directly the functional analysis method given in (Mahmudov, 2001a; Mahmudov and Denker, 2000; Mahmudov and Zorlu, 2003) for stochastic dynamical systems without delays, we obtain that the deterministic system (3) is approximately relatively controllable on $[0, T]$. However, taking into account that the state space for the deterministic dynamical system (3) is finite dimensional, i.e., exact and approximate controllability coincide (Klamka, 1991), we conclude that the deterministic dynamic system (3) is relatively controllable on $[0, T]$.

Remark 9. Let us observe that for a special case when the final time $T \leq h_{1}$, stochastic relative exact or approximate controllability problems in $[0, T]$ for the stochastic dynamical system with a delay in control (1) are reduced to the standard stochastic exact or stochastic approximate controllability problems for a stochastic dynamic system without delays in control (Klamka, 1991). From Theorem 1, two corollaries directly follow as special cases.

Corollary 1. (Mahmudov, 2001a; Mahmudov and Denker, 2000) Suppose that $T \leq h_{1}$. Then the stochastic dynamic control system (1) is stochastically relatively exactly controllable in $[0, T]$ if and only if

$$
\operatorname{rank}\left[B_{0}, A B_{0}, A^{2} B_{0}, \ldots, A^{n-1} B_{0}\right]=n .
$$

Corollary 2. (Mahmudov and Denker, 2000) The stochastic dynamic system without delays, i.e., $B_{j}=0, j=$ $1,2, \ldots, M$, is stochastically exactly controllable in any time interval if and only if the associated deterministic $d y$ namical system without delay is controllable.

Remark 10. Finally, it should be pointed out that using a general method given in the monograph (Klamka, 1991) for stochastically relatively approximately controllable dynamic systems it is possible to constuct admissible controls $u(t)$ defined for $t \in[0, T]$ and transferring a given initial state $x_{0}$ to the desired final state $x_{T}$ at time $T$.

\section{Systems with single delays}

In this section we shall consider a special case of the general semilinear stochastic systems described in Section 2, namely, systems with single point delays in control, described by the following differential state equation:

$$
\mathrm{d} x(t)=\left(A x(t)+B_{0} u(t)+B_{1} u(t-h)\right) \mathrm{d} t+\sigma \mathrm{d} w(t)
$$

for $t \in[0, T]$, with the initial conditions

$$
x(0)=x_{0} \in L_{2}\left(\Omega, F_{T}, \mathbb{R}^{n}\right) \text { and } u(t)=0
$$

for $t \in[-h, 0)$. Similarly as before, it is well known that for the given initial conditions (5) and any admissible control $u \in U_{a d}$, for $t \in[0, T]$ there exists a unique solution $x\left(t ; x_{0}, u\right) \in L_{2}\left(\Omega, F_{t}, \mathbb{R}^{n}\right)$ of the linear stochastic differential state equation (4), which can be represented in the following integral form:

$$
\begin{aligned}
& x\left(t ; x_{0}, u\right) \\
& =\exp (A t) x_{0} \\
& \quad+\int_{0}^{t} \exp (A(t-s))\left(B_{0} u(s)+B_{1} u(s-h)\right) \mathrm{d} s \\
& \quad+\int_{0}^{t} \exp (A(t-s)) \sigma \mathrm{d} w(s) .
\end{aligned}
$$

Thus, taking into account the zero initial control for $t \in$ $[-h, 0]$, the solution for $t \in[0, h]$ has the following form (Klamka, 1991):

$$
\begin{aligned}
x\left(t ; x_{0}, u\right)= & \exp (A t) x_{0} \\
& +\int_{0}^{t} \exp (A(t-s)) B_{0} u(s) \mathrm{d} s \\
& +\int_{0}^{t} \exp (A(t-s)) \sigma \mathrm{d} w(s) .
\end{aligned}
$$


Moreover, for $t>h$ we have

$$
\begin{aligned}
x\left(t ; x_{0}, u\right)= & \exp (A t) x_{0} \\
& +\int_{0}^{t} \exp (A(t-s)) B_{0} u(s) \mathrm{d} s \\
& \left.+\int_{0}^{t-h} \exp (A(t-s-h)) B_{1} u(s)\right) \mathrm{d} s \\
& +\int_{0}^{t} \exp (A(t-s)) \sigma \mathrm{d} w(s)
\end{aligned}
$$

or, equivalently,

$$
\begin{aligned}
x\left(t ; x_{0}, u\right)= & \exp (A t) x_{0}+\int_{0}^{t-h}\left(\exp (A(t-s)) B_{0}\right. \\
& \left.+\exp (A(t-s-h)) B_{1}\right) u(s) \mathrm{d} s \\
& +\int_{t-h}^{t} \exp (A(t-s)) B_{0} u(s) \mathrm{d} s \\
& +\int_{0}^{t} \exp (A(t-s)) \sigma \mathrm{d} w(s) .
\end{aligned}
$$

Now, for a given $T>h$, taking into account the form of the integral solution $x\left(t ; x_{0}, u\right)$, let us introduce the following operators and sets (Klamka, 1991).

Introduce the linear bounded control operator $L_{T} \in$ $L\left(L_{2}^{F}\left([0, T], \mathbb{R}^{m}\right), L_{2}\left(\Omega, F_{T}, \mathbb{R}^{n}\right)\right)$ as

$$
\begin{aligned}
L_{T}= & \int_{0}^{T-h}\left(\exp (A(T-s)) B_{0}\right. \\
& \left.+\exp (A(T-s-h)) B_{1}\right) u(s) \mathrm{d} s \\
& +\int_{T-h}^{T} \exp (A(T-s)) B_{0} u(s) \mathrm{d} s .
\end{aligned}
$$

Then its adjoint linear bounded operator is $L_{T}^{*} \in$ $L_{2}\left(\Omega, F_{T}, \mathbb{R}^{n}\right) \rightarrow L_{2}^{F}\left([0, T], \mathbb{R}^{m}\right)$,

$$
L_{T}^{*} z=\left\{\begin{array}{r}
\left(B_{0}^{*} \exp \left(A^{*}(T-t)\right)\right. \\
+B_{1}^{*} \exp \left(A^{*}(T-t-h)\right) E\left\{z \mid F_{t}\right\} \\
\text { for } t \in[0, T-h], \\
B_{0}^{*} \exp \left(A^{*}(T-t)\right) E\left\{z \mid F_{t}\right\} \\
\text { for } t \in(T-h, T],
\end{array}\right.
$$

and the set of all states reachable from initial state $x(0)=$ $x_{0} \in L_{2}\left(\Omega, F_{T}, \mathbb{R}^{n}\right)$ in time $T>0$ using admissible controls,

$$
\begin{aligned}
R_{T}\left(U_{a d}\right)= & \left\{x\left(T ; x_{0}, u\right) \in L_{2}\left(\Omega, F_{T}, \mathbb{R}^{n}\right): u \in U_{a d}\right\} \\
= & \exp (A t) x_{0}+\operatorname{Im} L_{T} \\
& +\int_{0}^{T} \exp (A(T-s)) \sigma \mathrm{d} w(s) .
\end{aligned}
$$

Moreover, we introduce the concept of the linear controllability operator (Klamka, 1991; 1993; Mahmudov 2001a; Mahmudov and Denker, 2000) $C_{T} \in$
$L\left(L_{2}\left(\Omega, F_{T}, \mathbb{R}^{n}\right), L_{2}\left(\Omega, F_{T}, \mathbb{R}^{n}\right)\right)$, which is strongly associated with the control operator $L_{T}$ and is defined by the following equality:

$$
\begin{aligned}
C_{T}= & L_{T} L_{T}^{*} \\
= & \int_{0}^{T-h}\left(\exp (A(T-t)) B_{0} B_{0}^{*} \exp \left(A^{*}(T-t)\right)\right. \\
& +\exp (A(T-t-h)) B_{1} B_{1}^{*} \\
& \left.\quad \exp \left(A^{*}(T-t-h)\right)\right) E\left\{\cdot \mid F_{t}\right\} \mathrm{d} t \\
& +\int_{T-h}^{T} \exp (A(T-t)) B_{0} B_{0}^{*} \\
& \quad \exp \left(A^{*}(T-t)\right) E\left\{\cdot \mid F_{t}\right\} \mathrm{d} t .
\end{aligned}
$$

Finally, let us recall the $n \times n$-dimensional deterministic controllability matrix (Klamka, 1991),

$$
\begin{aligned}
& G_{T} \\
& =\int_{0}^{T-h}\left(\exp (A(T-t)) B_{0} B_{0}^{*} \exp \left(A^{*}(T-t)\right)\right. \\
& \left.\quad+\exp (A(T-t-h)) B_{1} B_{1}^{*} \exp \left(A^{*}(T-t-h)\right)\right) \mathrm{d} t \\
& +\int_{T-h}^{T} \exp (A(T-t)) B_{0} B_{0}^{*} \exp \left(A^{*}(T-t)\right) \mathrm{d} t .
\end{aligned}
$$

In the sequel, we study the relationship between the controllability concepts for the stochastic dynamical system (4) and the controllability of the associated deterministic dynamic system of the following form:

$$
\begin{aligned}
y^{\prime}(t)= & A y(t)+B_{0} v(t) \\
& +B_{1} v(t-h), \quad t \in[0, T],
\end{aligned}
$$

where the admissible controls $v \in L_{2}\left([0, T], \mathbb{R}^{m}\right)$.

For completeness, let us recall the following result concerning the relative controllability of the deterministic system (6).

Lemma 3. (Klamka, 1991) The following conditions are equivalent:

(i) The deterministic system (6) is relatively controllable on $[0, T]$.

(ii) The controllability matrix $G_{T}$ is nonsingular.

(iii) There holds

$$
\begin{gathered}
\operatorname{rank}\left[B_{0}, B_{1}, A B_{0}, A B_{1}, A_{2} B_{0}, A_{2} B_{1}, \ldots,\right. \\
\left.A_{n-1} B_{0}, A_{n-1} B_{1}\right]=n .
\end{gathered}
$$

Finally, using the general results given in Theorem 1 and the conditions presented in Lemma 2, we can formulate the following corollary, which is a necessary and sufficient condition for both exact and approximate relative controllability of the stochastic system (4). 
Corollary 3. The following conditions are equivalent:

(iv) The deterministic system (6) is relatively controllable on $[0, T]$.

(v) The stochastic system (4) is stochastically relatively exactly controllable on $[0, T]$.

(vi) The stochastic system (4) is stochastically relatively approximately controllable on $[0, T]$.

\section{Concluding remarks}

In the paper sufficient conditions for stochastic relative exact controllability for linear stationary finite-dimensional stochastic control systems with multiple constant point delays in control have been formulated and proved. These conditions extend to the case of one constant point delay in control which corresponds to known stochastic exact controllability conditions for dynamic control systems without delays recently published in the papers (Mahmudov, 2001; 2002; Mahmudov and Denker, 2000). Finally, it should be pointed out that using standard techniques presented in the monograph (Klamka, 1991) it is possible to extend the results given in this paper to more general nonstationary linear stochastic control systems with many time variable point delays in control. Moreover, extensions to stochastic absolute exact controllability and stochastic absolute approximate controllability in a given time interval are also possible.

\section{References}

Arapostathis, A., George, R.K. and Ghosh, M.K. (2001). On the controllability of a class of nonlinear stochastic systems, Systems and Control Letters 44(1): 25-34.

Bashirov, A.E. and Kerimov, K.R. (1997). On controllability conception for stochastic systems, SIAM Journal on Control and Optimization 35(3): 348-398.

Bashirov, A.E. and Mahmudov, N.I. (1999). On concepts of controllability for deterministic and stochastic systems, SIAM Journal on Control and Optimization 37(6): 1808-1821.

Ehrhard, M. and Kliemann, W. (1982). Controllability of stochastic linear systems, Systems and Control Letters 2(2): 145-153.

Fernandez-Cara, E., Garrido-Atienza, M.J., and Real, J. (1999).On the approximate controllability of a stochastic parabolic equation with multiplicative noise, Comptes Rendus de l'Académie des Sciences, Paris 328(1): 675-680.

Kim, J. U. (2004). Approximate controllability of a stochastic wave equation, Applied Mathematics and Optimization 49(1): 81-98.

Klamka, J. (1991). Controllability of Dynamical Systems, Kluwer Academic Publishers, Dordrecht.

Klamka, J. (1993). Controllability of dynamical systems-A survey, Archives of Control Sciences 2(3/4): 281-307.
Klamka, J. (1996). Constrained controllability of nonlinear systems, Journal of Mathematical Analysis and Applications 201(2): 365-374.

Klamka, J. (2000). Schauder's fixed point theorem in nonlinear controllability problems, Control and Cybernetics 29(3): 377-393.

Klamka, J. and Socha, L. (1977). Some remarks about stochastic controllability, IEEE Transactions on Automatic Control 22(6): 880-881.

Klamka, J. and Socha, L. (1980). Some remarks about stochastic controllability for delayed linear systems, International Journal of Control 32(5): 561-566.

Mahmudov, N.I. (2001a). Controllability of linear stochastic systems, IEEE Transactions on Automatic Control 46(5): 724-731.

Mahmudov, N.I. (2001b). Controllability of linear stochastic systems in Hilbert spaces, Journal of Mathematical Analysis and Applications 259(1): 64-82.

Mahmudov, N.I. (2002). On controllability of semilinear stochastic systems in Hilbert spaces, IMA Journal of Mathematical Control and Information 19(2): 363-376.

Mahmudov, N.I. (2003a). Controllability and observability of linear stochastic systems in Hilbert spaces, Progress in Probability 53(2): 151-167.

Mahmudov, N. I. (2003b). Approximate controllability of semilinear deterministic and stochastic evolution equations in abstract spaces, SIAM Journal on Control and Optimization 42(5): 1604-1622.

Mahmudov, N.I. and Denker, A. (2000).On controllability of linear stochastic systems, International Journal of Control 73(2): 144-151.

Mahmudov, N.I. and Zorlu, S. (2003). Controllability of nonlinear stochastic systems, International Journal of Control 76(2): 95-104.

Subramaniam, R. and Balachandran, K. (2002). Controllability of stochastic Volterra integrodifferential systems, $\mathrm{Ko}$ rean Journal on Computing and Applied Mathematics 9(2): 583-589.

Sunahara, Y., Kabeuchi, T., Asada, S., Aihara, S. and Kishino, K. (1974). On stochastic controllability for nonlinear systems, IEEE Transactions on Automatic Control 19(1): 49-54.

Sunahara, Y., Aihara, S. and Kishino, K. (1975). On the stochastic observability and controllability for nonlinear systems, International Journal of Control 22(1): 65-82.

Zabczyk, J. (1991). Controllability of stochastic linear systems, Systems and Control Letters 1(1): 25-31. 


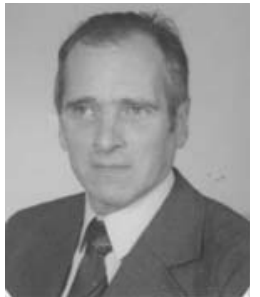

Jerzy Klamka received the M.Sc. and Ph.D. degrees in control engineering from the Silesian University of Technology in Gliwice, Poland, in 1968 and 1974, respectively. He also received the M.Sc. and Ph.D. degrees in mathematics from the University of Silesia in Katowice, Poland, in 1971 and 1978, respectively. In 1981 he received the postdoctoral degree in control engineering and in 1990 the professorial title in control engineering from the Silesian University of Technology in Gliwice, Poland. He has been a member of the American Mathematical Society (AMS) since 1976 and the Polish Mathematical Society (PTM) since 1982.
$\mathrm{He}$ is also a permanent reviewer for Mathematical Reviews (since 1976) and for Zentralblatt für Mathematik (since 1982). Since 2002 he has been a member of the Polish Academy of Sciences. In 1981 and 1991 he received the Polish Academy of Sciences awards. In 1978, 1982, 1990 and 2003 he was granted the awards of the Polish Ministry of Education. Moreover, in 1994 he received the Polish Mathematical Society award. In 1991 he published the monograph entitled Controllability of Dynamical Systems with Kluwer Academic Publishers, Dordrecht, The Netherlands. In the last 40 years he has published more than $100 \mathrm{pa}-$ pers in international journals. His major current interest is controllability theory for linear and nonlinear dynamical systems, and in particular the controllability of distributed parameter systems, dynamical systems with delays, and multidimensional discrete systems.

Received: 2 February 2008

Revised: 29 March 2008 
